\title{
PAPER
}

\section{Hypothesis on the pathophysiology of syringomyelia based on simulation of cerebrospinal fluid dynamics}

\author{
H S Chang, H Nakagawa
}

J Neurol Neurosurg Psychiatry 2003;74:344-347

See end of article for authors' affiliations

.....................

Correspondence to: Dr H S Chang, Department of Neurological Surgery, Aichi Medical University, 21 Yazako-Karimata, Nagakute-cho, Aichi-gun, Aichi 480-1195, Japan; chang@aichi-med-u.ac.jp

Received 14 June 2002 In final revised form

11 November 2002

Accepted

21 November 2002

\begin{abstract}
Objectives: Despite many hypotheses, the pathophysiology of syringomyelia is still not well understood. In this report, the authors propose a hypothesis based on analysis of cerebrospinal fluid dynamics in the spine.

Methods: An electric circuit model of the CSF dynamics of the spine was constructed based on a technique of computational fluid mechanics. With this model, the authors calculated how a pulsatile CSF wave coming from the cranial side is propagated along the spinal cord.

Results: Reducing the temporary fluid storage capacity of the cisterna magna dramatically increased the pressure wave propagated along the central canal. The peak of this pressure wave resided in the mid-portion of the spinal cord.

Conclusions: The following hypotheses are proposed. The cisterna magna functions as a shock absorber against the pulsatile CSF waves coming from the cranial side. The loss of shock absorbing capacity of the cisterna magna and subsequent increase of central canal wall pressure leads to syrinx formation in patients with Chiari I malformation.
\end{abstract}

$\mathrm{S}$ yringomyelia is a disease that produces fluid containing cavities in the parenchyma of the spinal cord. Most often it is associated with Chiari type I malformation with a herniation of cerebellar tonsils through the foramen magnum. In 1958, Gardner described his hypothesis on the pathophysiology of this disease ${ }^{1}$; he considered that an obstruction of the cerebrospinal fluid (CSF) outflow from the fourth ventricle diverts the CSF pulse waves into the central canal. His hypothesis was thus called the "water-hammer theory". Based on his theory, suboccipital craniectomy with plugging of the obex, obstructing the opening of the central canal, became common. Recently, however, most surgeons agree that bony decompression of the foramen magnum together with some form of dural decompression is all that is required to treat this type of syringomyelia..$^{3-6}$ Gardner's theory is difficult to reconcile with this operation because, if the obex is not plugged, CSF pulses will be propagated down the central canal.

Ball and Dayan ${ }^{7}$ proposed that the CSF enters the syrinx through the enlarged Virchow-Robin space in the spinal cord. Although several other authors agree with this hypothesis, their opinions differ about specific mechanisms. Ball and Dayan hypothesised that the CSF is driven into the VirchowRobin space when the spinal subarachnoid pressure is increased during coughing or straining. They postulated that this increased pressure is caused by a one way valve-like mechanism at the craniovertebral junction, which blocks the upward CSF movement. Heiss et $a l^{8}$ thought that the piston-like movement of the herniated tonsils is responsible for producing downward pulse waves in the subarachnoid space, which contribute to the downward progression of the syrinx. Stoodley et $a l,{ }^{90}$ on the other hand, considered that increased compliance of the spinal subarachnoid space increases the arterial pulse dependent CSF flow through the Virchow-Robin space.

In this report, we took a different approach to evaluate this problem. We theoretically analysed the dynamics of the pressure wave propagating through the spinal subarachnoid space. For this purpose, we built an electric circuit model of the spinal CSF pathway, based on the standard analysis technique of the arterial pulse wave. ${ }^{11}$ We then applied a sudden increase of voltage on the cranial side of this model, simulating the CSF wave coming from the cranial side. By examining this model, we could analyse how the pressure wave propagated along the spinal cord.

\section{METHODS}

Figure 1 shows our electric circuit model superimposed on the actual anatomical structures. The CSF pathways are broken up into multiple nodal points starting from the fourth ventricle and prepontine cistern, followed by obex and cisterna magna, and then by nine nodal points and finally the lumbar theca. (The number of nodal points is arbitrary.) We represented the compliance (or the temporary fluid storage capacity) of the cisterna magna with a capacitor named $\mathrm{C}_{\text {cist }}$. In the spine, two pathways of CSF, namely the central canal and the extramedullary subarachnoid space, are represented as series of multiple resistors. To represent the compliance of the spinal cord and the dural sac, we inserted two arrays of capacitors: one between these two CSF pathways, and the other between the subarachnoid CSF pathway and the ground. Thus, the capacitors between the central canal and the subarachnoid space $\left(\mathrm{C}_{1}\right.$ to $\mathrm{C}_{9}$ in fig 1) represent the elasticity (compliance) of both the central canal wall and the spinal cord. Similarly, the capacitors between the subarachnoid space and the ground $\left(\mathrm{D}_{1}\right.$ to $\mathrm{D}_{9}$ in fig 1) represent the elasticity of both the dural sac and the root sleeves. Table 1 shows the values of the parameters that we used for the calculations in this study.

We could solve this electrical diagram with the standard analysis technique of a linear system. After setting the initial voltage accumulated in $\mathrm{C}_{1}$ to $\mathrm{C}_{9}$ to zero, and that in $\mathrm{C}_{\text {cist }} \mathrm{D}_{1}$ to $\mathrm{D}_{9}$ and $\mathrm{C}_{\text {thec }}$ to 100 , we applied a step increase of voltage of 500 simultaneously on the two cranial leads. Mathematically, this problem is reduced to solving a set of linear ordinary differential equations with the voltages accumulated in all the capacitors taken as unknown variables. These equations were solved on a personal computer using a software package: Mathematica version 4.0 (Wolfram Research, Champaign, IL, USA). 


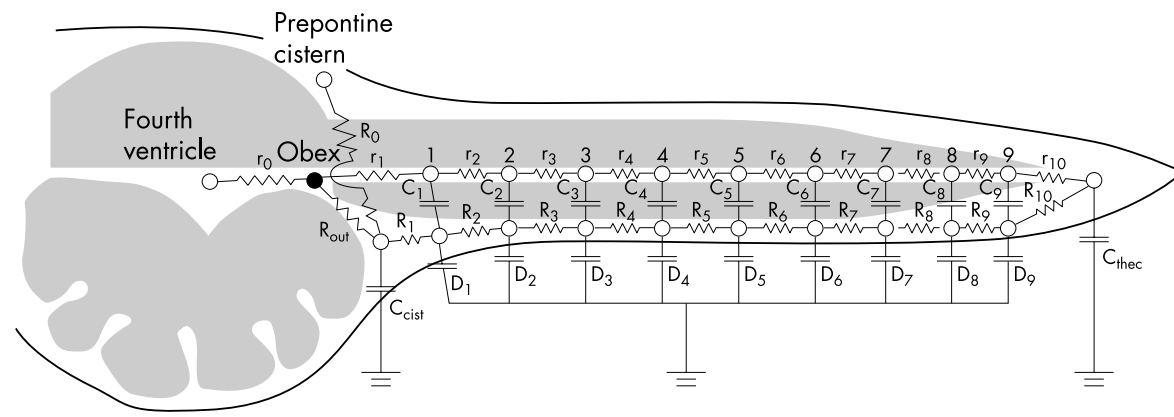

Table 1 The values of the resistors and capacitors in our diagram

\begin{tabular}{ll}
\hline Parameter & Value \\
\hline$r_{1}$ to $r_{9}$ & 100 \\
$R_{1}$ to $R_{9}$ & 0.2 \\
$C_{1}$ to $C_{9}$ & 0.1 \\
$D_{1}$ to $D_{9}$ & 600 \\
$r_{0}$ & 0.2 \\
$R_{\text {out }}$ & 0.0002 \\
$R_{0}$ & 0.02 \\
$C_{\text {cist }}$ & 600000 \\
$C_{\text {thec }}$ & 6000000 \\
\hline
\end{tabular}

We then examined the effect of changing the temporary fluid storage capacity (or compliance) of the cisterna magna. Because reduced compliance at the cisterna magna is equivalent to reduced capacitance of $\mathrm{C}_{\text {cist }}$ (fig 1 ) in our model, we calculated the response of our circuit with three different values of $\mathrm{C}_{\text {cist }}$ : the original value, one tenth of the original value, and one hundredth of the original value.

To simulate the effect of syringo-subarachnoid shunting, we inserted a small resister valued 0.2 bypassing the capacitor $\mathrm{C}_{4}$ in figure $\mathrm{l}$ in addition to the reduced value of $\mathrm{C}_{\text {cist }}$ to one hundredth of the original value.

\section{RESULTS}

The time course of the pressure wave propagated along the central canal in response to a sudden increase of voltage on the two cranial leads is shown in figure 2. In biological terms, the voltage accumulated in these capacitors corresponds to the pressure difference between inside and outside the spinal cord at each nodal point, a positive value of the voltage indicating higher pressure inside. In the following figures, the horizontal coordinate shows the nodal points ( 1 to 9 in fig 1 ) in our model, the left side being the cranial side and the right side being the caudal side. The time course of the accumulated voltage at each nodal point is shown as indicated in the figures starting from the time of application of the pulsatile wave at time zero. Figure 2A, B, and C show the responses of the system with three different values of capacitance at the cisterna magna $\left(C_{\text {cist }}\right.$ in fig 1$)$. As the capacitance of $C_{\text {cist }}$ was decreased, there was a dramatic increase of the central canal wall pressure. The peak of this pressure increase was at the mid-portion of the spinal cord.

The result of the simulation of syringo-subarachnoid shunting is shown in figure 3. In this case, we set the value of $\mathrm{C}_{\text {cist }}$ at one hundredth of the original value. In this setting, the pressure response is increased as in figure 2C. We placed an electrical shunt at the nodal point 4 (fig l) between the central
Figure 1 A diagram showing our electric circuit model of the cerebrospinal fluid dynamics in the spine. The diagram is superimposed on actual anatomical structures. The upper row of resistors $\left(r_{1}\right.$ to $\left.r_{10}\right)$ represents the central canal, and the lower row $\left(R_{1}\right.$ to $\left.R_{10}\right)$ represents the spinal subarachnoid space. The capacitor $\mathrm{C}_{\text {cis }}$ represents the compliance of the cisterna magna, and $\mathrm{C}_{\text {thec }}$ that of the lumbar thecal sac. The capacitors $C_{1}$ to $C_{9}$ represent the elasticity of the central canal wall and the cord parenchyma. The capacitors $D_{1}$ to $D_{9}$ represent the elasticity of the dura and nerve roots. The digits 1 to 9 show the number of the nodal points.

canal and the subarachnoid space. As shown in figure 3, this shunting effectively reduced the central canal wall pressure, although it was less effective on the cranial side.

\section{DISCUSSION}

Our results showed that reduced compliance at the craniovertebral junction increased the pressure wave propagated through the spinal cord. This phenomenon is analogous to the Windkessel model of the cardiovascular system. ${ }^{11}$ In the Windkessel model, the aorta functions as an elastic tube with temporary fluid storage capacity. This temporary fluid storage capacity is understood with an analogy to a Windkessel-a reservoir of a water pump of a fire engine (fig 4). When the aorta becomes stiff and loses this temporary fluid storage capacity, the arterial pulse wave becomes steep resulting in systolic hypertension. ${ }^{11}$ Likewise, our model provides a hypothesis explaining why the constriction at the craniovertebral junction causes the formation of syrinx in the spinal cord. When the temporary fluid storage capacity of the cisterna magna is reduced as in Chiari type I malformation, the pressure wave propagated through the central canal is increased. In other words, we can think that the cisterna magna normally functions as a shock absorber, which absorbs the pulse pressure of the CSF coming from the cranial side. If this shock absorbing capacity is lost, because of the overcrowding of the posterior fossa in Chiari I malformation, the CSF pressure wave propagated along the central canal is markedly increased; it will then lead to leakage of CSF into the parenchyma, which precedes the formation of syrinx. Thus, we may consider that our hypothesis is a modified version of Gardner's water-hammer theory. The difference is that our theory can explain why extradural decompression at the craniovertebral junction without intradural procedure can reduce the size of the syrinx in the spinal cord.

Our hypothesis is better in some regards than the other hypotheses presupposing the role of the Virchow-Robin space. Firstly, it can explain the phenomenon of syringomyelia with no tonsillar herniation, and why foramen magnum decompression is effective in reducing the size of the syrinx. ${ }^{12}$ Tubbs et $a l^{13}$ showed that these patients had moderately small posterior fossae. Our hypothesis can explain this phenomenon well, because we can assume that the temporary fluid storage capacity at the cisterna magna is reduced in such patients because of the mild overcrowding of the posterior fossa. On the other hand, other hypotheses have difficulty explaining this phenomenon, because they require either a one way valve mechanism or a piston-like movement of the cerebellar tonsils, which are less likely to occur in those patients with no Chiari malformation. Secondly, our model is compatible with the fact that syringo-subarachnoid shunting is effective in reducing the size of the syrinx. Although the syringosubarachnoid shunting is known to have poor long term 

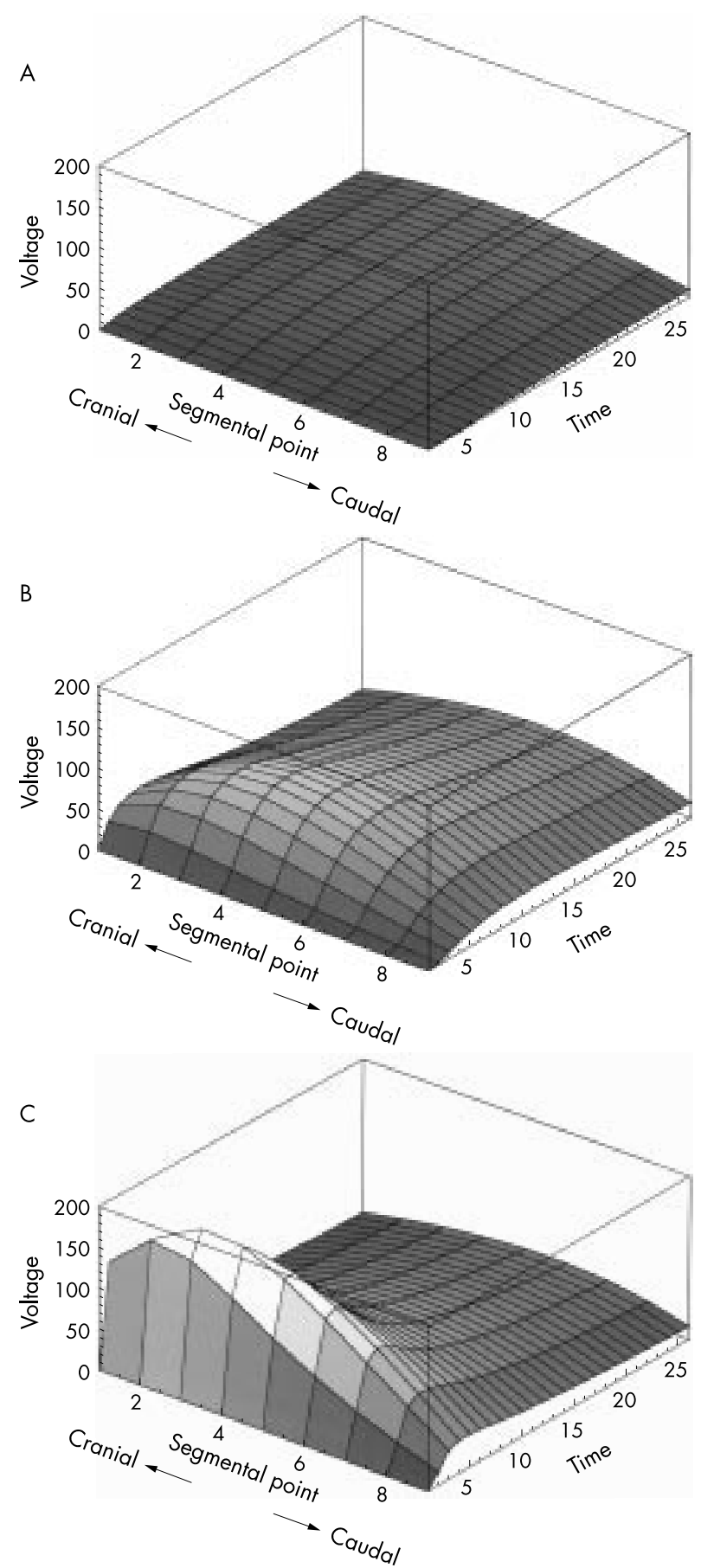

Figure 2 Three dimensional plots showing the wall pressure of the central canal (represented as the voltage of the capacitors $C_{1}$ to $C_{9}$ ) after application of a step input on the cranial leads at time 0 . The voltage at each nodal point 1 to 9 in figure 1 is plotted. The value of $\mathrm{C}_{\text {cist }}$ is set at the original value in $\mathrm{A}$, one tenth of the original value in $B$, and one hundredth of the original value in $C$ respectively.

results mainly because of obstruction of the tube, it is usually effective in short-term. ${ }^{36}$ If we assume that the CSF enters from the subarachnoid space to the syrinx through the Virchow-Robin space, we cannot easily explain this phenomenon; a larger conduit made by a syringo-subarachnoid shunt will, simply interpreted, rather aggravate the syrinx.

The weak point of our hypothesis might be that it assumed the patency of the central canal, or an existence of some other channel connecting the fourth ventricle and the syrinx. Only in about $14 \%$ of the patients with syringomyelia associated

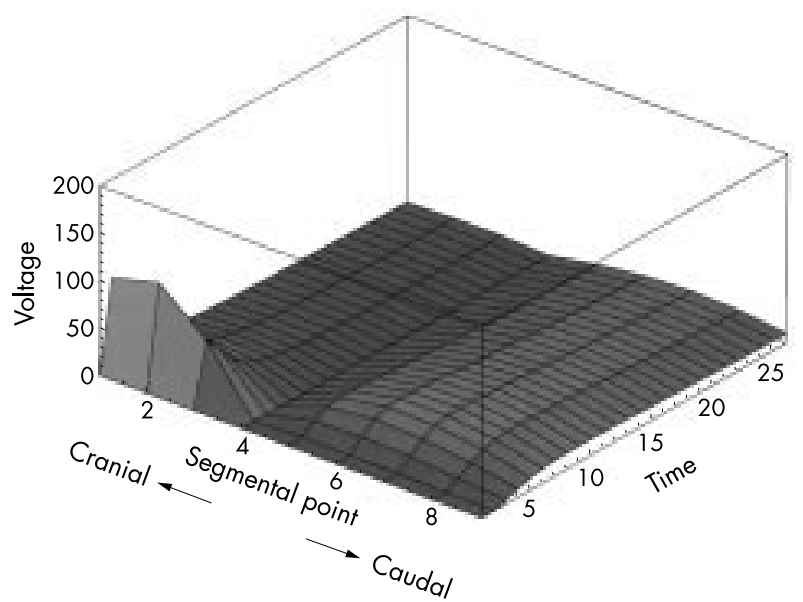

Figure 3 Simulation of syringo-subarachnoid shunt. The same plot as figure $2 \mathrm{C}$ except for an insertion of a small resistor shunting the capacitor $\mathrm{C}_{4}$ in figure 1 simulating the syringo-subarachnoid shunt. The increased wall pressure response seen in figure $2 \mathrm{C}$ is much reduced.

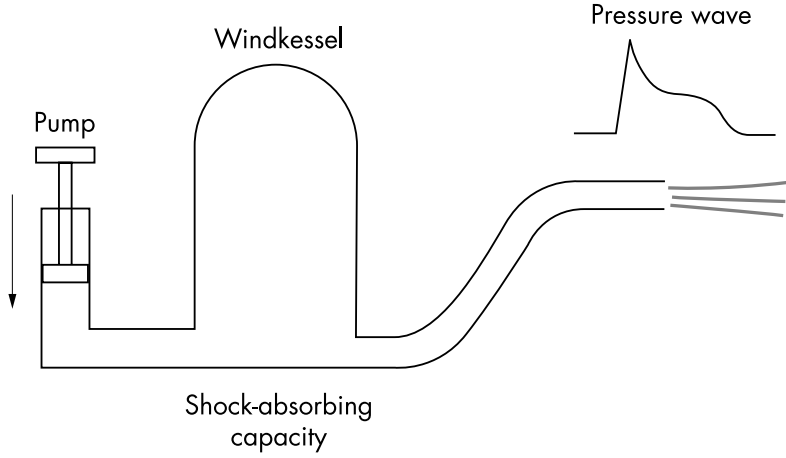

Figure 4 Cisterna magna functions like a Windkessel of a fire engine' s water pump. If it becomes "stiff" and loses its temporary fluid storage capacity as in Chiari I malformation, the pressure wave propagated along the central canal is increased, leading to syrinx formation.

with Chiari type I malformation, was the connection between the syrinx and the fourth ventricle demonstrated on MRI. ${ }^{14}$ Also, cadaver studies showed that the central canal was obliterated in a large proportion of the human population as the age increased. ${ }^{15}{ }^{16}$ We can make several arguments concerning this point. Firstly, to say the least, our theory can explain the pathophysiology in those $14 \%$ of patients who have communicating syringomyelia associated with Chiari type I malformation. Secondly, MRI may not be able to demonstrate the connection between the syrinx and the fourth ventricle. There is some evidence that the current resolution of MRI cannot detect the normal sized central canal. ${ }^{17}{ }^{18}$ In that case, we may have larger proportion of patients with communicating syringomyelia that can be explained by our theory. Actually, Milhorat et al found in their pathological study that, in $42 \%$ of syringomyelia associated with Chiari I malformation, the syrinx was rostrally communicating with the patent central canal. ${ }^{19}$ Thirdly, our model showed that if there was stenosis of the central canal at some point, the pressure wave was markedly increased immediately rostral to that stenotic point (data not shown). If we postulate that this increased pressure wave ruptures the ependyma of the central canal, and drives the formation of syrinx inside the parenchyma, our theory does not necessarily contradict the necropsy finding of progressive obliteration of the central canal in human. Fourthly, even if the central canal is obliterated in a large percentage of the elderly human population, it does not necessarily mean that the 
same thing happens in the patients with Chiari type I malformation. In those patients, our results suggest that the comparatively small volume of the posterior fossa causes increased pressure waves along the central canal; this increased pressure wave may then prevent the normal process of occlusion of the central canal. Fifthly, if we closely observe our model, we find that the patency of the central canal is not absolutely required. Our model only assumed some fluid channel inside the spinal cord, and this fluid channel may well be the Virchow-Robin space.

Our theory may also be criticised because we used rather arbitrary values on the resistors and capacitors of our circuit. However, we were only interested in the qualitative behaviour of our circuit, not the quantitative determination of the amplitude and the time course of the pressure waves. The qualitative behaviour of our circuit was basically unchanged with different sets of parameters that we tested. Our study is theoretical in nature; we believe that a theory that can elegantly explain the actual phenomena can be justified as far as its assumptions do not absolutely contradict the experimental findings.

Our theory is important in the clinical perspective. Even though we have a standard surgical approach to patients with syringomyelia associated with Chiari malformation, it is not satisfactory if we do not clearly understand why it works. Better understanding of the pathophysiology will certainly improve the results of our surgery. In addition to syringomyelia associated with Chiari type I malformation, we have other types of syringomyelia such as that associated with adhesive arachnoiditis. Our theory was also effective in explaining the pathophysiology of syringomyelia associated with adhesive arachnoiditis, thus providing a unifying theory on those two types of syringomyelia. ${ }^{20}$ We believe that our theory can serve as a working hypothesis for further clinical and experimental works.

\section{ACKNOWLEDGEMENT}

We thank Professor Marie Oshima, PhD at Institute of Industrial Science, University of Tokyo, and Professor Kotaro Minato, PhD at Nara Institute of Science and Technology for their assistance in the technical aspect of this study.

Authors' affiliations

H S Chang, H Nakagawa, Department of Neurological Surgery, Aichi Medical University, Japan

Competing interests: none declared.

\section{REFERENCES}

1 Gardner WJ, Angel J. The cause of syringomyelia and its surgical treatment. Cleve Clin Q 1958:25:4-8.

2 Gardner WJ. Hydrodynamic mechanism of syringomyelia: its relationship to myelocele. J Neurol Neurosurg Psychiatry 1965;28:247-59.

3 Hida K, Iwasaki Y, Koyanagi I, et al. Surgical indication and results of foramen magnum decompression versus syringosubarachnoid shunting for syringomyelia associated with Chiari I malformation. Neurosurgery 1995; 37:673-9.

4 Milhorat TH, Chou MW, Trinidad EM, et al. Chiari I malformation redefined: clinical and radiographic findings for 364 symptomatic patients. Neurosurgery 1999;44:1005-17.

5 Isu T, Sasaki $H$, Takamura $H$, et al. Foramen magnum decompression with removal of the outer layer of the dura as treatment for syringomyelia occurring with Chiari I malformation. Neurosurgery 1993;33:844-50.

6 Iwasaki Y, Abe H. Surgical treatment for syringomyelia. No Shinkei Geka 1996;24:709-16.

7 Ball MJ, Dayan AD. Pathogenesis of syringomyelia. Lancet 1972;ii:799-801.

8 Heiss JD, Patronas N, DeVroom HL, et al. Elucidating the pathophysiology of syringomyelia. J Neurosurg 1999:91:553-62.

9 Stoodley MA, Brown SA, Brown CJ, et al. Arterial pulsation-dependent perivascular cerebrospinal fluid flow into the central canal in the sheep spinal cord. J Neurosurg 1997;86:686-93.

10 Stoodley MA, Gutschmidt B, Jones NR. Cerebrospinalfluid flow in an animal model of noncommunicating syringomyelia. Neurosurgery 1999;44:1065-76.

11 Glasser SP. On arterial physiology, pathophysiology of vascular compliance, and cardiovascular disease. Heart Disease 2000;2:375-9.

12 Iskandar BJ. The resolution of syringohydromyelia without hindbrain herniation after posterior fossa decompression. J Neurosurg 1998;89:212-16.

13 Tubbs RS, Elton S, Grabb P, et al. Analysis of the posterior fossa in children with the Chiari 0 malformation. Neurosurgery 2001;48:1050-5

14 Milhorat TH, Johnston WD, Miller Jl, et al. Surgical treatment of syringomyelia based on magnetic resonance imaging criteria. Neurosurgery 1993;33:535-6.

15 Milhorat TH, Kotzen RM, Anzil AP. Stenosis of central canal of spinal cord in man: incidence and pathological findings in 232 autopsy cases. $J$ Neurosurg 1994:80:716-22.

16 Yasui K, Hashizume Y, Yoshida M, et al. Age-related morphologic changes of the central canal of the human spinal cord. Acta Neuropathol 1999;97:253-9.

17 Petit-Lacour MC, Lasjaunias P, Iffenecker C, et al. Visibility of the central canal on MRI. Neuroradiology 2000;42:756-61.

18 Beuls E, Gelan J, Vandersteen M, et al. Microanatomy of the excised human spinal cord and the cervicomedullary junction examined with high-resolution MR imaging at 9.4 Tesla. ANNR Am J Neuroradiol 1993:14:699-707.

19 Milhorat TH, Capocelli AL, Jr, Anzil AP, et al. Pathological basis of spinal cord cavitation in syringomyelia: analysis of 105 autopsy cases. $J$ Neurosurg 1995;82:802-12

20 Chang HS, Nakagawa H. Physical analysis of CSF dynamics in the spine. In: Tamaki N, Batzdorf U, Nagashima T, eds. Syringomyelia. Current concepts in pathogenesis and management. Tokyo: Springer, 2001:69-72. 